\title{
The DELPHI Silicon Tracker - Barrel part
}

\author{
V. Chabaud
}

Presented at the 5th International Conference on Advanced Technology and Particle Physics, Como, Italy, 5-11 October, 1996 


\title{
The DELPHI Silicon Tracker - Barrel part
}

\author{
V. Chabaud
}

Presented at the 5th International Conference on Advanced Technology and Particle Physics, Como, Italy, 5-11 October, 1996 


\title{
The DELPHI Silicon Tracker - Barrel part
}

\author{
V. Chabaud, DELPHI Collaboration ${ }^{\mathrm{a}}$ \\ ${ }^{a}$ CERN, CH-1211 Geneva 23, Switzerland
}

A new central tracker, with larger angular acceptance, has been installed early 1996 inside the DELPHI detector. The tracker consists of 3 sub-detectors: a Si microstrip barrel detector having 3 layers with $R \phi$ and $z$ read-out, surrounded by a $\mathrm{Si}$ macropixel detector, surrounded in turn by a $\mathrm{Si}$ "ministrip" detector. The overall angular acceptance is $11^{\circ}<\theta<169^{\circ}$. We shall mostly restrict to the barrel detector and review its design, construction, performances and geometrical alignment.

\section{GENERAL DESCRIPTION}

Until 1995 the DELPHI experiment used a barrel vertex detector[1] consisting of 3 layers of $\mathrm{Si}$ microstrip detectors (Fig. 1). We used to call the 3 layers Outer, Inner and Closer respectively. Outer and Closer layers were consisting of 24 modules each with double-sided read-out, Inner layer of 24 modules with single-sided read-out only. The mean radii of the 3 layers were 10.9 , 9.0 and $6.3 \mathrm{~cm}$, and the polar angle coverage was going down to $\theta \sim 40^{\circ}$ for Outer and Inner layers and $\theta=25^{\circ}$ for Closer layer. This angular acceptance was clearly insufficient for the new physics expected at LEP2 energies and it had been decided in 1992 to launch an upgrade program[2]. Many Laboratories have contributed to this project ${ }^{1}$.

Fig. 1 shows a perspective view of the new Silicon Tracker. It consists of 3 sub-detectors: an extended 3-layer Si microstrip barrel, surrounded at larger(smaller) $\theta$ by two rings of 38 overlapping Si macropixel detectors on each side, surrounded in turn at even larger(smaller) $\theta$ by two rings of 12 overlapping $\mathrm{Si}$ "ministrip" modules ${ }^{2}$ on each side. The overall angular acceptance is now $11^{0}<\theta<169^{\circ}$.

The macropixel detectors have pixels of $330 \times$ $330 \mu \mathrm{m}^{2}$ and provide space points with a precision of about $90 \mu \mathrm{m}$. Note that the first ring of

\footnotetext{
${ }^{1}$ Bratislava, CERN, Grenoble, Helsinki, Karlsruhe, Krakow, Liverpool, Ljubljana, CPPM Marseille, Milano, LAL Orsay, Oxford, Padova, CdF Paris, LPNHE Paris, Prague, RAL, Roma, Uppsala, Vienna, Wuppertal.

${ }^{2}$ One ministrip module $=$ two back-to-back detectors .
}
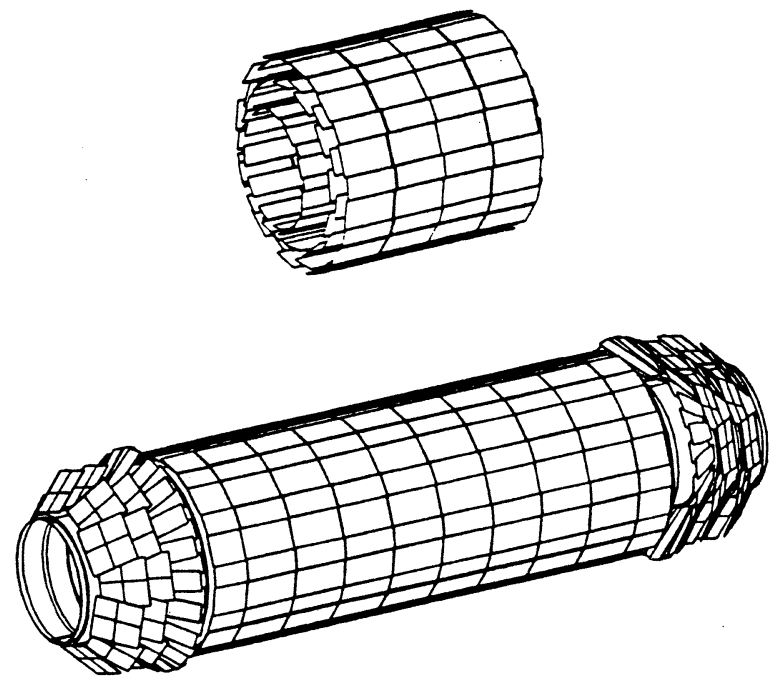

Figure 1. Top: perspective view of the 1995 vertex detector; bottom: perspective view of the new Silicon Tracker.

macropixel detectors on either side are not visible in Fig. 1 as they are tightly packed inside the Inner layer of the barrel ${ }^{3}$.

Each ministrip module consists of 2 detectors with single sided readout glued back to back. The 2 detectors have orthogonal strips with a pitch of $100 \mu \mathrm{m}$, every second strip being readout. A stereo angle of $4^{0}$ has been introduced between the adjacent 2 rings on each side to reduce re-

\footnotetext{
${ }^{3}$ Due to production delays both internal rings but only half an external ring were installed in 1996.
} 
construction ambiguities. The ministrip modules provide space points with a precision of 20 to $30 \mu m[3]$.

In the rest of this paper we shall restrict ourselves to the barrel detector ${ }^{4}$.

\section{DESIGN AND CONSTRUCTION OF THE BARREL}

\subsection{Geometrical features}

The three Outer, Inner and Closer layers have mean radii of $11.0,9.1$ and $6.5 \mathrm{~cm}$ and sensitive lengths of $47.6,46.3$ and $27.3 \mathrm{~cm}$ respectively. Their angular acceptance goes down to $\theta=24^{\circ}, 21.5^{\circ}$ and $25^{\circ}$ respectively. The three layers consist of 24,20 and 24 modules each and they measure both the $R \phi$ and the $z$ coordinates of particle hits, except the central part $\left(37^{0}<\theta<143^{\circ}\right)$ of Inner layer which has only an $R \phi$ readout. The modules of all layers are overlapping each other at least $10 \%$, so that 3 to $6 R \phi$ and 2 to $6 z$ coordinates can be measured per track. These overlaps are an essential tool for the alignment procedure.

\subsection{Mechanical features}

The Silicon tracker consists of two independent half-shells. All modules of the barrel are supported by two complex aluminum end rings inside which a flow of cooling water removes the $440 \mathrm{w}$ of heat released by the readout electronics. The two end rings are also connected by a thin cylindrical carbon fibre sheet to reinforce the mechanical structure and to minimize a possible overall twist deformation of the Silicon tracker during transport and insertion. Individual modules are stiffened by gluing along their length a thin $\Omega$-shaped beam made out of Kevlar and carbon fibre. Much care was taken to minimize the amount of material seen by particles. It is equivalent to $0.027 \mathrm{X}^{0}$ at $\theta=90^{\circ}$ (half of it being at larger $R$, which is less detrimental) and it has been severely reduced in the range $\theta=20^{\circ}-40^{\circ}$ compared to the previous vertex detector.

\footnotetext{
${ }^{4}$ See in the same Volume the contribution by $W$. Adam on Performance of the DELPHI VFT ministrip detector.
}

\subsection{Electrical features}

More details will be given in the following sections. Here we mention only features common to all layers and all modules.

The $n^{+}$implants are separated with field plates or $p^{+}$blocking electrodes, depending upon the producer of the detectors. The $p^{+}$and $n^{+}$implants of all detectors are AC-coupled to readout metal lines through integrated capacitors. The $z$ coordinate signals appearing on these metal lines are routed toward the far end of the corresponding half-module via a second layer of metal lines orthogonal to the first one. The $R \phi$ or $z$ readout metal lines of the two or four detectors forming a half-module are joined together by daisychaining and read out by chips at the far end of the half-module. Upon the arrival of a trigger all analog signals on these chips are sent to a number of Fastbus SIROCCO units (developed at CERN) located in the counting room, for digitization, pedestal calculation, noise calculation and zero suppression by DSP56001 processors.

\subsection{Details on Closer layer modules}

The Closer layer consists of 24 modules which have been reused from the 1995 vertex detector (the final upgrade had already been anticipated for this layer). Each module consists of 4 detectors with double-sided readout, manufactured by SINTEF (Oslo). The detectors are $2.1 \mathrm{~cm}$ wide, and 7.9 or $6.1 \mathrm{~cm}$ long for the central and extreme ones respectively. Fig. 2 shows a sketch of the two sides of a half-module.

The p-side of the first or second detector provides $R \phi$ coordinates and includes 768 diodes, every second one being read out ( $50 \mu \mathrm{m}$ pitch). The corresponding readout metal lines are bonded to the inputs of $3 \mathrm{MX} 6$ readout chips mounted on a beryllium oxide hybrid.

The $n$-side of the first or second detector provides $z$ coordinates and includes 1152 and 384 implants respectively which are all read out. As shown in Fig. 2 the readout pitch increases toward the hybrid so that the charge deposited by a hit be spread over a comparatively small number of strips, in order to keep a reasonable signal-tonoise ratio on each strip. To reduce the number 


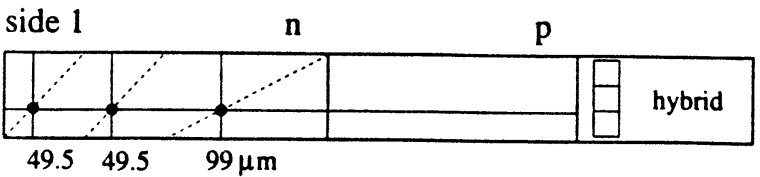

sisce $\mathrm{p} \quad \mathrm{n}$

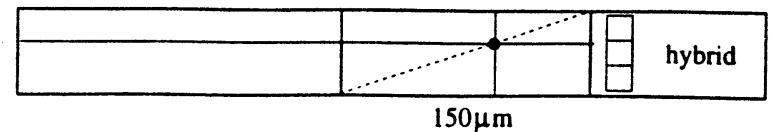

Figure 2. Sketch of the two sides of a half-module of Closer layer.

of electronic channels $3 \mathrm{n}$-strips of the first detector are connected (multiplexed) to one of the 384 metal lines of the second metal layer, which are bonded to 3 other MX6 chips. This multiplexing results in little additional ambiguity in the pattern recognition of tracks in DELPHI.

We should now explain the odd $n / p$ or $p / n$ look of a half-module. Thanks to the AC-coupling the readout lines on either side of a detector are at the same potential and it is possible to flip one detector with respect to the other and to bond the $\mathrm{n}$-side readout lines of one to the $\mathrm{p}$-side lines of the other, and vice versa. This so-called "flipped module design" has two advantages. It first equalizes the noise on the two sides of a detector, which results in an increase of the signalto-noise ratio on the critical n-side (with more stray capacitance). In addition the polarity of the read out signal tags which detector produced it.

Note that in Fig.2 we have represented with horizontal and vertical lines the readout metal lines involved when one single track hits the halfmodule. We have also represented with diagonal lines the alignment of the connections between the two layers of metal lines on the $n$-side.

\subsection{Details on Inner layer modules}

The Inner layer consists of 20 modules having 8 detectors each. They have been built out of the previous 4-detector modules of the 1995 Outer layer after ungluing them in the middle and inserting and gluing 4 new detectors with single- sided readout. All detectors have been manufactured by Hamamatsu. They are $3.3 \mathrm{~cm}$ wide and 5.75 or $5.96 \mathrm{~cm}$ long. Fig. 3 shows a sketch of the two sides of a half-module. The flipped module design has been adopted as for Closer layer.

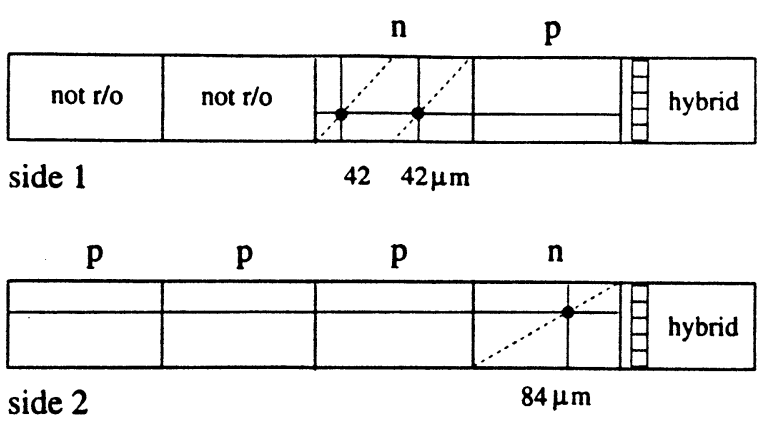

Figure 3. Sketch of the two sides of a half-module of Inner layer.

The p-side of all detectors provides $R \phi$ coordinates and includes 1280 diodes, every second one being read out $(50 \mu m$ pitch). The corresponding 640 readout metal lines are bonded to 5 MX6 readout chips. The n-side of the extreme 2 detectors provides $z$ coordinates and includes 1280 and 640 implants respectively which are all read out with multiplexing by 5 other MX6 chips.

Because of the reuse of these 2 detectors for a different $\theta$ range the readout pitch $(42$ and $84 \mu \mathrm{m})$ is not optimum and results in clusters of 9 to 14 strips with a smaller signal-to-noise ratio per strip. This effect however leads to no inefficiency after an appropriate adjustment of the cluster search algorithm in the corresponding DSP56001 processors.

\subsection{Details on Outer layer modules}

The Outer layer consists of 24 modules with 8 detector pairs each. Since multiple scattering is less detrimental at larger $R$ the solution of pairs of single-sided detectors glued back-to-back has been adopted for economical reasons. The detectors have been manufactured by Hamamatsu $(R \phi)$ and SINTEF $(z)$. They are $3.3 \mathrm{~cm}$ wide and 
$5.96 \mathrm{~cm}$ long. Fig. 4 shows a sketch of the two sides of a half-module.

\section{$\mathrm{R} \phi$ side}

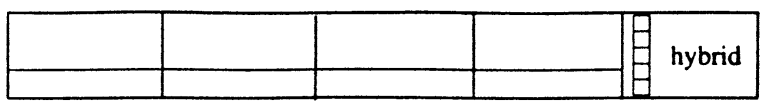

$\mathrm{Z}$ side

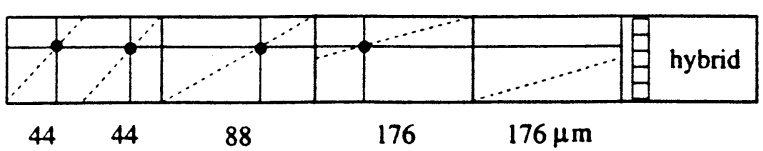

Figure 4. Sketch of the two sides of a half-module of Outer layer.

The $R \phi$ side of the 4 pairs includes 1280 diodes, every second one being readout $(50 \mu \mathrm{m}$ pitch). The 640 readout metal lines are bonded to 5 TRIPLEX readout chips developed at Orsay. The diodes on the $z$ side of the 4 pairs have a pitch of $44 \mu \mathrm{m}$ and readout pitches as indicated in the Figure. All diodes are read out for the first detector, every second one for the second detector, while every first and second strips are connected and read out and every third and 4th strips are skipped for the last two detectors.

In the design of the $z$ side we had to accept a small charge loss on the not connected strips. Despite that we shall see in the following Section that the signal-to-noise ratio per cluster is still very large.

\section{PERFORMANCE AND ALIGNMENT OF THE BARREL}

The Silicon Tracker was inserted inside DELPHI at the beginning of May. Collecting a sample of cosmic rays allowed us to check the cabling and the read out and to align roughly the two halfshells. At the end of June LEP began operating at the $Z^{0}$ peak and delivered an integrated luminosity of $0.5 p b^{-1}$, intended for calibration and alignment of the LEP experiments. In particular about $400 Z^{0}$ decays into muon pairs were collected inside the acceptance of the barrel detec- tor. From July 8th to August 18th LEP operated at the new higher energy of $161 \mathrm{GeV}$ and delivered an integrated luminosity of $12 p b^{-1}$.

During this period a strict temperature stability of the Silicon Tracker was maintained (better than $1^{\circ}$ ) resulting in a very good geometrical stability of the detector, both internally and externally with respect to the other tracking detectors of DELPHI.

The hit efficiency of the barrel was found to be very good, with only a few problematic halfmodules (not supplied or very noisy).

The $Z^{0}$ data allowed us to measure precisely the signal-to-noise ratio of the 3 layers. We found the Landau distributions peaking at 31,16 and 17 for $R \phi$ clusters in the 3 Outer, Inner and Closer layers respectively, and peaking at 22,22 and 13 for the $z$ clusters.

\subsection{Alignment of the barrel}

The alignment of the barrel (as well as of the macropixel and ministrip detectors) is performed in two stages: an optical and mechanical survey first, followed by an alignment using particle tracks.

In the survey stage all modules are measured in turn by a high precision optical system (Mondo camera), which provides the position of all strips on the two sides of a module with respect to two high precision reference spheres fitted on one side of the two hybrids. After assembling all modules into half-shells, a 3D survey is made with a high precision mechanical probe (Poli station), which provides the relative positions of all modules of all layers of each half-shell. The overall precision of the survey is about $25 \mu \mathrm{m}$.

The alignment by software uses tracks from $Z^{0}$ decays: tracks of muon pairs, tracks passing through the overlap region of two adjacent modules and tracks from hadronic decays measured as $3 R \phi$ coordinates and 2 or $3 z$ coordinates. The aims are: to refine the internal alignment of either half-shell, to align the two half-shells with respect to each other and to align externally the full barrel with respect to the other tracking detectors of DELPHI. The target alignment precision is 3 to $5 \mu m$ say.

The principle is very simple. Tracks through over- 
laps allow us to align the Outer layer, muon pair tracks allow to align the Closer layer with respect to the Outer one and hadronic tracks allow to align the Inner layer with respect to the other two layers.

The practice is more difficult[4]:

- Given the lightness of the mechanical structure, a small overall twist of the barrel might develop during transport and insertion. This overall twist might even induce a local twist of the individual modules. Both effects must be measured and parametrized.

- We also found that individual modules might develop a small radial bending, not necessarily the same for all modules of a given layer. This effect is small for the Closer layer, but the amplitude of the deformation might be as large as 50 to $100 \mu m$ in the middle of a module of Inner or Outer layer. This effect is related to some humidity difference between the survey and the insertion inside DELPHI but is not fully understood yet. It must also be measured and parametrized.

- One has also to take into account a possible and variable acolinearity and momentum unbalance of the LEP beams when using muon pairs for alignment. These effects have to be measured by the LEP engineers and are not available in real time. - Finally one has to cope with the much smaller integrated luminosity delivered at the $Z^{0}$ peak in 1996. This year we collected only 400 muon pairs for alignment instead of several thousands in the previous years.

\subsection{Barycentric shift}

A puzzling effect is found when studying and parametrizing radial bending. We look at the residual of the $R \phi$ hits in the Closer layer of the tracks passing through two adjacent modules and plot its distribution as a function of the average $z$ coordinate of the two hits (Fig.5).

With a perfect alignment we should see a flat distribution about the horizontal axis with a r.m.s equal to the intrinsic precision times $\sqrt{2}$. What we see actually consists of two effects: a bell shape apparently superimposed on two small jumps at $z \simeq \pm 8 \mathrm{~cm}$. The bell shape demonstrates the small radial bending of Closer layer modules. As regards the two small jumps we checked that

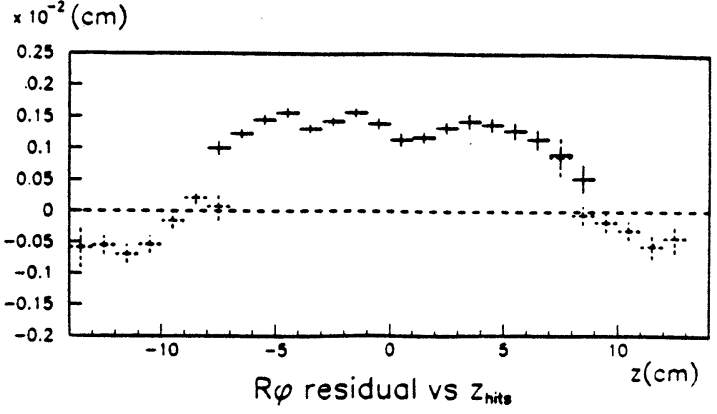

Figure 5. Distribution of the residual of the two $R \phi$ hits of a track passing through two overlapping modules of Closer layer as a function of the average $z$ coordinate of the hits.

they occur precisely at the boundaries between the flipped detectors of the modules ${ }^{5}$. Given the high precision of the survey of individual modules (much better than the overall survey precision of $25 \mu \mathrm{m}$ ) the simplest explanation would be that the distribution of holes created by a particle does not correspond exactly to the mid-plane of the detectors and this offset can be observed here because it goes in opposite directions in the two flipped detectors. We have observed the same effect for the Inner modules and also with the 1995 vertex detector. The barycentric shift is of the order of 10 to $20 \mu \mathrm{m}$ toward the p-side of the detectors $\left(4 \times \sin 7.5^{\circ}\right.$ times the observed jumps for Closer layer).

It is more difficult to check if the same effect exists for the electrons created together with holes by a particle (we found some indication with the 1995 vertex detector also toward the p-side). It would be interesting to confirm these observations with a dedicated beam test experiment. One would need a telescope of 4 or 5 standard detectors (no flipped modules), very precisely spaced, having alternate orientations $(\mathrm{n} / \mathrm{p}, \mathrm{p} / \mathrm{n}, \mathrm{n} / \mathrm{p}, \ldots)$, traversed by the beam under symmetric angles (20 to $30^{\circ}$ say) with respect to the normal.

\footnotetext{
${ }^{5}$ The jump at $z \simeq 8 \mathrm{~cm}$ is less sharp than the one at $z \simeq$ $-8 \mathrm{~cm}$. The smoothing-out is due to the $4 \mathrm{~mm}$ odd/even staggering of the Closer layer modules.
} 


\subsection{Tracking precision}

The tracking precision is the convolution of intrinsic hit precision, alignment precision and multiple scattering. It is not easy to disentangle the first two components since remaining misalignments might affect some parts of the detector more than others or affect some variables more than others.

The best indicator of the overall tracking precision of the barrel is given by the missed distance between the two muon tracks from $Z^{0}$ decays, from which one derives the precision on the asymptotic (negligible multiple scattering) impact parameter (I.P.). From the small sample of $Z^{0}$ decays into muon pairs used for alignment, as well as the second sample collected in october, we have found the following results.

I.P. $(R \phi)$ for all $\theta=26 \mu m(21 \mu m)$

I.P. $(z)$ for $\theta \simeq 90^{\circ}=48 \mu m(36 \mu m)$

I.P. $(z)$ for $|\cot \theta|<1=65 \mu m(71 \mu m)$

I.P. $(z)$ for all $\theta=86 \mu m(-)$.

The numbers between brackets give for comparison the results obtained with the 1995 vertex detector.

Note that these results should still be taken as preliminary.

\section{CONCLUSION}

The new DELPHI Silicon Tracker has been successfully installed and operated in 1996. The barrel detector was found to have a very good efficiency and a tracking precision close to that of the 1995 vertex detector. After preliminary alignment and error calibration it has been able to contribute satisfactorily to tracking and b-tagging in the analysis of the LEP2 data collected at 161 and $172 \mathrm{GeV}$.

\section{REFERENCES}

1. V. Chabaud et al., The DELPHI Silicon Strip Microvertex Detector with Double Sided Readout, CERN-PPE/95-86 and Nucl. Instr. and Meth. A368 (1996) 314.

2. The DELPHI Collaboration, Proposal for the new DELPHI Very Forward Tracker, note DELPHI 93-52 GEN 146.
3. A. Andreazza et al., The DELPHI Very Forward Tracker for LEP200, Nucl. Instr. and Meth. A367 (1995) 198.

4. V. Chabaud et al., Alignment of the DELPHI vertex detector, note DELPHI 95-177 MVX 10. 